\title{
The influence of high efficiency engines and hybrids on exhaust systems
}

Increased efficiency of the combustion process itself and low losses in the Engine, lead to lower temperatures in the exhaust line. Combined with the exhaust gas energy recovery as well as the hybridization of the drivetrain, this temperature decrease will require additional efforts for the exhaust gas aftertreatment in future. Current technologies like SCR with urea could only be used in future with additional heating elements or will need to change to different catalysts or gaseous Ammonia, to keep the current efficiency and conversion rates. Catalyst and filter elements with ultra-low backpressure creating additional new challenges for the correct and robust diagnostics of these aftertreatment components and all emission relevant parts and thresholds. New technologies are needed like the direct measurement of the DPF soot loading with radio frequencies or $\mathrm{NH}_{3}$ sensors to precisely control the ammonia slip for high conversion rates of SCR catalysts.

Key words: high efficiency engines, low exhaust temperature, $\mathrm{NH}_{3}$ sensor, low backpressure DPF

\section{Introduction}

The increasing efficiency of combustion engines, together with the hybridization of drivetrains, is influencing the aftertreatment system including its control strategies and diagnostics. The reduction of mechanical losses due to use of new lubricants and the cylinder reduction, e.g. from 6 to 5 cylinders for commercial vehicle diesel engines, combined with optimized direct injection strategies, increased injection pressure and advanced fuels, allows the next generation of combustion engines a tremendous increase in efficiency and therefor, also a reduction in $\mathrm{CO}_{2}$ emissions.

Increased efficiency in the combustion process itself and low losses in the Engine, lead naturally to lower temperatures in the exhaust line. Combined with the exhaust gas energy recovery as well as the hybridization of the drivetrain, this temperature decrease will require additional efforts for the exhaust gas aftertreatment in future. Current technologies like SCR with urea could only be used in future with additional heating elements or will need to change to gaseous ammonia, to keep the current efficiency and conversion rates.

New catalyst and filter elements with ultra-low backpressure contribute to further $\mathrm{CO}_{2}$ reduction leading to new challenges to perform a correct and robust diagnostics of these aftertreatment components.

To fulfill future emission laws, regarding emission limits and diagnostics of the aftertreatment system, new Sen- sors like the direct measurement of the DPF soot loading with radio frequencies or $\mathrm{NH}_{3}$ sensors to precisely control the ammonia slip for high conversion rates of SCR catalysts are needed

\section{Current systems (diesel example)}

Today's engines are not operating at the most fuel efficient operating points. This is done to reduce the formation of raw emissions, mostly nitrogen oxides. Also, to reduce Particles and to heat the exhaust system, post injections late in the combustion or after combustion are used, leading to a higher temperature of the exhaust gas. The loss of efficiency created with this measure is accepted in general for passenger car applications. For larger engines, like commercial vehicles and non-road mobile machinery (e.g. heavy construction equipment), an additional HC-dosing unit is mounted in the exhaust line to inject fuel in front of the diesel oxidation catalyst (DOC). The fuel is then oxidized in the DOC, leading to a further increase of the exhaust temperature. Prerequisite for this methodology is that the temperature of the DOC is above 200 degrees Celsius. If this temperature cannot be reached, the use of a separate external burner unit to heat up the aftertreatment is needed.

Figure 1 shows an example of a current diesel aftertreatment system. It consists of a diesel oxidation catalyst, diesel particle filter and a SCR-catalyst for $\mathrm{NO}_{\mathrm{x}}$ reduction. The potential ammonia slip is treated with a cleanup catalyst combined with the SCR-catalyst.

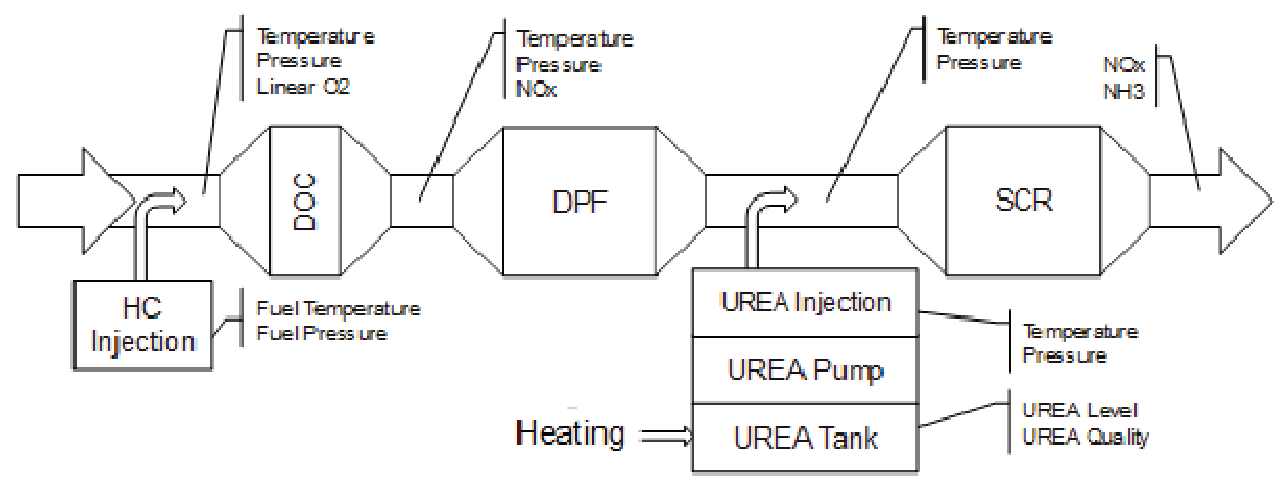

Fig. 1. Current diesel exhaust system example [1] 
This aftertreatment systems has a conversion rate depending on the temperature of the exhaust gas and the catalysts. For SCR systems based on urea, the maximum conversion rate is between 200 and 300 degrees Celsius. Below or above this temperature window, this system has lower conversion rates leading to a non-fulfillment of actual emission norms.

The efficiency of the diesel particle filter (DPF) depends on the used materials and technology. Based on the chosen technology, the DPF needs a temperature window either for continuous conversion or to start the regeneration of a loaded filter element. In medium to high load points of the engine, the exhaust gas temperature today is high enough to heat the diesel oxidation catalyst to his light off temperature. This allows the use of additional heating with a post injection in passenger cars, and in addition with a $\mathrm{HC}$ dozer for commercial vehicles.

The issue arising here is, that as the efficiency of an engine increase, the exhaust gas temperature of the engine will decrease. This will lead to lower conversion rates and issues to regenerate the diesel particle filter. Once the diesel oxidation catalyst is not getting heated enough, the whole exhaust aftertreatment process is at stake.

\section{High efficiency engines}

Besides emissions, the next generation of combustion's engines will be optimized also for $\mathrm{CO}_{2}$ emissions. The efficient combustion of this engines lead to a lower exhaust gas temperature. In addition, exhaust gas energy recovery systems will further reduce the exhaust temperature. This systems convert energy of the exhaust gas into electricity, or transferring it to the coolant water of the engine. Another trend to increase the efficiency is downsizing of the engines and the reduction of the number of cylinders. This lowers the exhaust volume and therefore also the amount of energy available. Furthermore, due to cylinder reduction and new lubricants, the friction of the engines is reduced. The reduction of this engine losses lead to the situation, that in the already critical area of low load with low exhaust gas temperature, the exhaust gas energy is further reduced, as less fuel is needed to keep the engine idling.

Besides classical engine design optimization, hybridization is even for commercial vehicles an upcoming methodology, to increase the overall powertrain efficiency [2]. Energy is recovered by using the electrical motors for regenerative braking. This recovered energy is then used to accelerate the vehicle using an additional electrical engine. This additional engine, assists the combustion engine in the dynamic phase of an acceleration, which is emission critical. Also, according the relation of the vehicle weight and the available electrical torque, vehicles can drive fully electrical for some time, or eradicating the Engine idle with a start/stop functionality completely. These measures are leading to a reduced load, or even to a complete standstill for the combustion engine. As a result, less energy in the exhaust gas for the exhaust system is available.

In future applications, the overall design of the combustion engine including its exhaust system needs to take care of this issue. Engineers must find an optimal operating strategy to balance high efficiency of the combustion en- gine and low $\mathrm{CO}_{2}$ against the emissions and the needs of the exhaust system.

\section{At the cross road - integrated or standalone}

Because of the trend to high efficiency engines, we will see the further integration of the exhaust system into the combustion engine for passenger cars and smaller engines. Oxidation catalyst, $\mathrm{NO}_{\mathrm{x}}$ storage catalyst or even the threeway catalyst will be mounted close, or even at the engine inside the engine bay. This will minimize the temperature losses of the exhaust gas before reaching the catalysts or filter elements. The temperature management of the whole system needs to guarantee the needed operating temperature, depending on the operating point of the engine and the produced exhaust gas.

Also, hybrids with increasing capability of driving fully electric laying down extra requirements on this management. Before the combustion engine can take over with medium or full power, there needs to be a heating strategy implemented to get the exhaust system to the required conversion performance. This means, that also the hybrid driving strategy needs to consider the operational status of the exhaust system.

From a controls or operating strategy standpoint of view, this is an issue in classical automotive engineering as the automotive industry is mostly component driven. Distributed functionality based on a system engineering approach to optimize and solve this issue is rare.

For larger engines like trucks, busses or non-road mobile machinery, the exhaust system can be considered as a standalone. Based on the expected emissions, volume stream and allowed back pressure, the active elements can be designed and a feed forward control can best established. Such a stand-alone unit will then request a certain concentration of hydrocarbons and an according temperature for the incoming volume stream and use own heating units, like a $\mathrm{HC}$ dozer and oxidation catalysts, electrical heater or separate burner units for further temperature control in the exhaust system.

\section{The end of urea?}

Most currently used $\mathrm{NO}_{\mathrm{x}}$ catalyst systems are using a liquefied solution of urea. This solution is the Injected into the exhaust pipe in front of the SCR catalyst. Besides issues with crystallization at the Injector and aging of the solution, a liquid urea solution needs a certain exhaust gas temperature to create the necessary vapor to release the needed ammonia gas. Lower temperatures limit this process and therefore the distance between urea injector and SCRcatalyst needs to be increased to ensure a proper vaporization. Below 200 degrees Celsius most urea based systems have not sufficient conversion rates to fulfil legal emission limits.

An interesting alternative to this systems was developed by the Danish company AMINEX [3] where ammonia gas is directly stored in a salt. This salt is the used in cartridges in the vehicle, enabling a safe handling of the stored ammonia. The release of the needed ammonia for the aftertreatment system is reached by heating the salt in the cartridges. 
Such a system can operate at much lower temperatures as urea based systems as they directly deliver ammonia gas. This makes them an interesting alternative for future aftertreatment systems beyond EURO 6/EURO VI and TIER 4.

\section{Missing: systems engineering}

Current automotive development is mostly component driven. Major OEM's are structured into engine departments and aftertreatment departments which reflects in the current function- and electronic control unit architecture. For further optimization, systems engineering on vehicle, functional and architectural level will become more and more important. At least in the controls structure of passenger cars, there should be no longer a system combustion engine accompanied by a system aftertreatment. In future, there will be a need to design this as one control system, where combustion engine, aftertreatment components and electrical components are sub systems of the overall driveline. Based on the actual status and capability of all components, it will create the maximum torque with highest possible efficiency.

\section{Future aftertreatment systems}

Three areas need to be improved for future exhaust systems like the example shown in Figure 2 are the control strategy, diagnostic and the catalysts themselves. As a first step to keep up the conversion rates of today's exhaust gas systems, we will see new sensors and sensor technology. As an example, the usage of a $\mathrm{NH}_{3}$ sensor, will allow closed loop control of the ammonia slip. This will enable the exhaust system to operate with the maximum urea quantities also on lower temperatures, leading to higher overall conversion rates as with feed forward control strategies.

Also, the urea quality sensor will assist in a more precise calculation of the needed dosing quantity to treat the current $\mathrm{NO}_{\mathrm{x}}$ in the exhaust stream. Together with the $\mathrm{NH}_{3}$ sensor, this results in a more robust and precise dosing strategy with feedback control as we have today.

Using this capability of the SCR system to operate always with its maximum conversion rate, future combustion engines can increase the production of raw emissions like $\mathrm{NO}_{\mathrm{x}}$, leading to better fuel performance and therefore $\mathrm{CO}_{2}$ reduction.

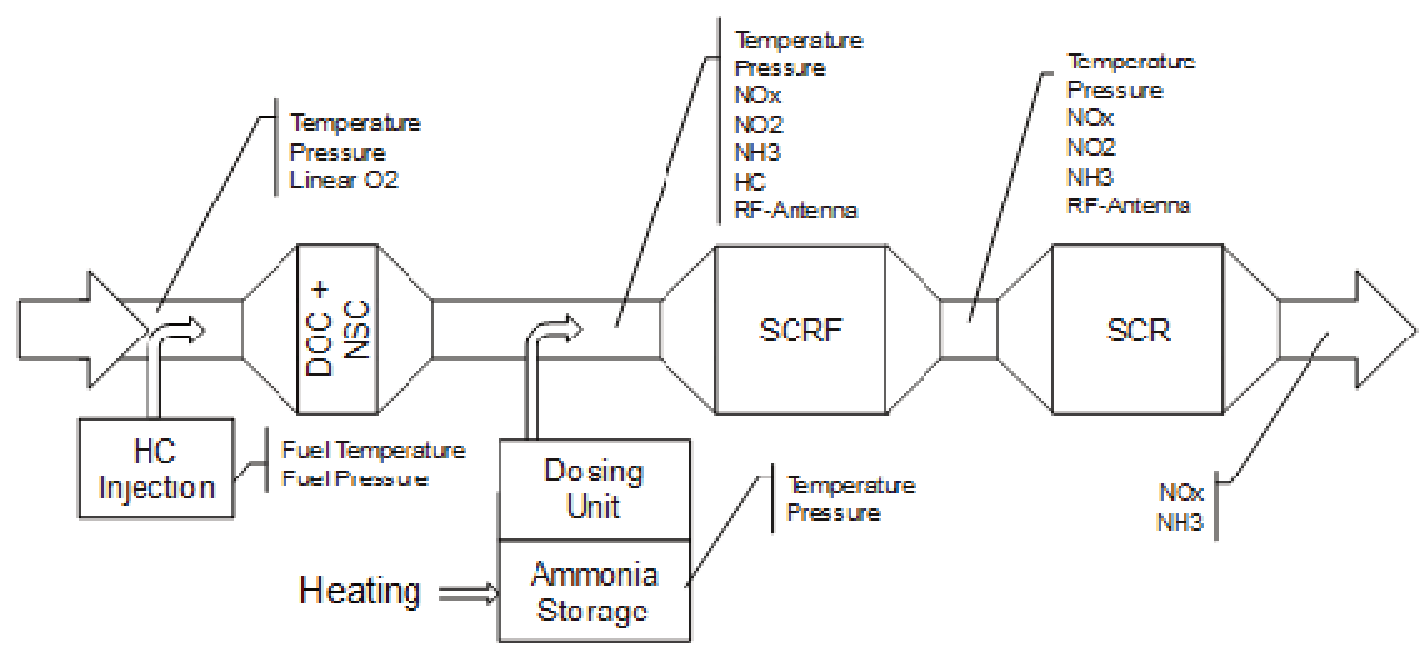

Fig. 2. Future diesel exhaust system example [1]

To establish a feedback control for the regeneration of the diesel particle filter, new sensors like the radio frequency based sensor [4] can be used. With this measurement principle, for the first time in the vehicle, the loading of the filter can be measured and the regeneration will only be performed if necessary. The regeneration based on a maximum time interval, to prohibit damages, is no longer necessary in such systems. This lead overall to a reduced number of regenerations and therefore to lower $\mathrm{CO}_{2}$ emissions.

To increase the robustness of the aftertreatment diagnostic, especially the diagnostic of the particle filter a particle sensor after the filter can be used. With this sensor, it will be possible to indirectly detect cracks in the filter ceramics due to measurement of the increased mass of particles after the filter element. current diagnostic strategies reaching their detection precision with the introduction of ultra-low backpressure filter ceramics. Diagnosing a crack in this components by pressure drop, is no longer possible.

This is an issue for diagnostics, as a major principle and rule of On Board Diagnostics II is: "If you can't diagnose it, you can't use it". Hence this sensor will open the usage of a variety of low back pressure components.

Regarding future catalysts, we will see active coating for $\mathrm{NO}_{\mathrm{x}}$ conversion also on the particle filter itself. This measure aims to increase the available active surface for $\mathrm{NO}_{\mathrm{x}}$ conversion (SCR on filter, SCRF). Also, the diesel oxidation catalyst will most likely be combined with a $\mathrm{NO}_{\mathrm{x}}$ storage catalyst. This will further increase the $\mathrm{NO}_{\mathrm{x}}$ conversion and storage capability, which is needed to reach an effective aftertreatment system shortly after engine start while the exhaust system itself is still heating up. Once the operation temperature is reached; the $\mathrm{NO}_{\mathrm{x}}$ storage can be regenerated.

Because of the SCRF element replacing of a classical diesel particle filter, the ammonia gas will be introduced into the exhaust gas stream before the SCRF and not like today after the diesel particle filter. This will increase the complexity of the $\mathrm{NO}_{\mathrm{x}}$ conversion control strategy. An additional $\mathrm{NH}_{3}$ sensor between the SCRF and the SCR 
catalyst will most likely be used to enable a feed back control for this configuration.

\section{Summary}

The overall trend to higher efficiency engines and lower $\mathrm{CO}_{2}$ emissions, lead to lower temperatures in the exhaust gas aftertreatment system.

This creates the demand for new technologies, like catalysts and additional Sensors, to provide the needed conver- sion efficiency and conversion rates to fulfill future emission norms and $\mathrm{CO}_{2}$ targets.

Future control strategies will utilize new and additional sensors, like $\mathrm{NH}_{3}$ sensor and RF-sensor, to implement closed loop control for regeneration of the particle filter and control of the $\mathrm{NO}_{\mathrm{x}}$ conversion rate.

To enable the usage of ultra-low backpressure components, todays diagnostic strategies need to be extended. This can be done by utilizing of urea quality sensors and particle mass sensor into the aftertreatment system.

\section{Nomenclature}

DOC diesel oxidation catalyst

DPF diesel particle filter

$\mathrm{HC}$ hydrocarbon

$\mathrm{NH}_{3}$ ammonia

NSC $\mathrm{NO}_{\mathrm{x}}$ storage catalyst

$\mathrm{NO}_{2}$ nitrogen oxide

\section{Bibliography}

[1] WEBER, M. Exhaust aftertreatment of diesel engines and its OBD. 3rd International Specialist Conference: SENSORS for Exhaust Gas Cleaning and $\mathrm{CO}_{2}$ Reduction. Leipzig, Germany.

[2] SHUTTLEWORTH, J. Volvo trucks tests hybrid powertrain for longhaul transport in concept truck. SAE Article. articles.sae.org/15330.

$\mathrm{NO}_{\mathrm{x}}$ nitrogen oxides

$\mathrm{O}_{2} \quad$ oxygen

$\mathrm{RF}$ radio frequence

SCR selective catalytic reduction

SCRF SCR on filter

Matthias Weber, Dipl.-Ing. - Managing Director and Founder of Roben Automotive.

e-mail: Matthias.Weber@roben-automotive.com
[3] JOHANNESSEN, T., SCHMIDT, H., SVAGIN, J. et al. Ammonia storage and delivery systems for automotive $\mathrm{NO}_{\mathrm{x}}$ aftertreatment. SAE Technical Paper. 2008, 2008-01-1027.

[4] RAGAller, P., SAPPOK, A., BROMBERG, L. et al. Particulate filter soot load measurements using radio frequency sensors and potential for improved filter management. SAE Technical Paper. 2016, 2016-01-0943.

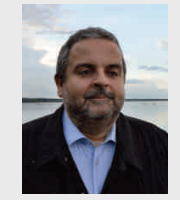

